\title{
La communication organisationnelle et numérique : formation en mutation, profession en construction
}

Sylvie P. Alemanno, Maître de Conférences-HDR, Université de Nice Sophia-Antipolis, Sylvie.parrinialemanno@unice.fr 


\section{Résumé}

Le dispositif de l'apprentissage universitaire en communication paraît faire émerger de façon singulière la problématique de la construction des compétences du groupe professionnel des communicants. Nous supposons que ce type de dispositif pour une formation en communication à un niveau master soulève la problématique du primat du terrain dans la formation des compétences spécifiques à la communication des organisations. En d'autres termes, une appropriation de la formation des étudiants, notamment apprentis, par les professionnels, instaurés tuteurs d'apprentissage par le dispositif, pourrait-elle se faire aux dépends de la formation académique ? De récentes études montrent que la définition du groupe professionnel des communicants est fortement soumise aux mutations organisationnelles dues pour partie au numérique. Nous explorons, suivant une démarche compréhensive, cette écologie organisationnelle en cours de complexification et les effets d'une communication essentialisée par les professionnels dont le numérique est devenu l'instrument princeps. Nous avons procédé par questionnaires et entretiens auprès des étudiants et professionnels attachés à la formation par apprentissage d'un Master en communication à l'Université de Nice Sophia Antipolis.

Mots-clefs : communicant, communication numérique, compétence, dispositif, apprentissage, groupe professionnel

\footnotetext{
Abstract

The university learning device in communication seems to emerge in a singular way the issue of the construction skills of the professional group of communicators. We assume that this type of device for communication training at a master level raises the issue of the primacy of the field in the formation skills specific to the communication of the organizations. In other words, ownership of the training of students, including apprentices, by professionals, learning tutors introduced by the device, it could be done at the expense of academic training? Recent studies show that the definition of " professional groups " of communicators is highly subject to organizational changes due in part to digital. We explore, following a comprehensive approach, this organizational ecology being complexity and the effects of essentialised communication professionals whose digital has become the instrument originator. We conducted by questionnaires and interviews with students and professionals committed to the apprenticeship of a Master in Communication at the University of Nice Sophia Antipolis.

Keywords: communicator, digital communication, competence, device, learning, professional group
} 
Communiquer est inhérent à toutes les activités professionnelles en organisation. De ce fait, faciliter, organiser, rechercher, partager l'information pour assurer une stratégie communicationnelle utile s'est instaurée en de véritables métiers différemment déclinés selon l'expertise technique. La professionnalisation qui a tardé à poser la profession de communicant (ou communicateur) (Benoit, 2000), poursuit son évolution dans le même mouvement que celle du web, web social et réseaux socio-numériques, devenus les incontournables supports de la profession. Or des tensions demeurent entre les besoins des organisations et des professionnels et les apports de la formation académique en communication. Ces tensions paraissent porter sur une opérationnalité immédiate des étudiants sortis de ces filières. Logiquement, des écarts apparaissent entre les attentes managériales souvent fondées sur une idéologie attachée au numérique et les compétences techniques et stratégiques des futurs communicants. En effet, ces écarts se creusent entre les pratiques de terrain des professionnels qui procèdent d'une adaptation accélérée à un contexte organisationnel concurrentiel et les démarches réflexives à propos d'une communication dite organisante proposée par l'enseignement universitaire. Ils semblent perdurer et incitent l'enseignant-chercheur à questionner la reconnaissance accordée par les professionnels à la professionnalisation des futurs communicants et par conséquence à leur formation, leurs formateurs et la structure en l'occurrence universitaire qui les reçoit.

Ces tensions hétérogènes interrogent donc les dispositifs de formation des communicants et leurs agencements, leurs acteurs (formateurs, professionnels en poste, étudiants) et les contenus de formations. Le dispositif de l'apprentissage universitaire en communication paraît faire émerger de façon singulière la problématique de la construction des compétences du groupe professionnel des communicants. Nous supposons que le dispositif de l'apprentissage universitaire en communication soulève la problématique du primat du terrain dans la formation des compétences spécifiques à la communication des organisations. En d'autres termes, une appropriation de la formation des étudiants, notamment apprentis, par les professionnels, instaurés tuteurs d'apprentissage par le dispositif se ferait-elle aux dépends de la formation académique? Dès lors, quelle définition induit-elle du « groupe professionnel » des communicants.

Le courant de la sociologie des professions, notamment en France, et plus précisément la sociologie des groupes professionnels offrent de sérieuses pistes de recherche pour comprendre la formation identitaire d'un groupe professionnel et du contenu de leur travail (savoir-faire des professionnels). Nous croisons pour cette recherche, les données des travaux en SIC qui ouvrent sur la problématique spécifique de la professionnalisation et des compétences communicationnelles afférentes. En effet, la notion de compétence développée par les sociologues, se trouve à redéfinir lorsqu'elle se situe dans le domaine de la communication compte tenu de la variété des professions qui la composent et des pratiques en 
transformations de professionnels. Enfin pour comprendre la problématique de la construction du groupe professionnel des communicants à partir du dispositif d'apprentissage des futurs communicants, il est pertinent de mobiliser la notion de dispositif en référence aux travaux de Foucault et Agemben. Cette notion ouvre des pistes pour saisir le processus de professionnalisation des communicants et la dynamique des acteurs pris dans la complexité de l'environnement organisationnel.

Nous explorons, suivant une démarche compréhensive, cette écologie organisationnelle en cours de complexification et les effets d'une communication essentialisée par les professionnels dont le numérique est devenu l'instrument princeps. Nous avons procédé par questionnaires et entretiens auprès des étudiants et professionnels attachés à la formation par apprentissage d'un Master en communication à l'Université de Nice Sophia Antipolis.

\section{Eléments de théorie : construction d'un groupe de professionnels}

La notion de profession est généralement étudiée par la sociologie des professions qui s'est penchée sur celle de groupes professionnels (Dubar, Tripier, 1998, Bourdoncle, 2000, Champy, 2012). Elle éclaire les préoccupations sur la profession et les métiers des communicants. Dubar appelle " "groupe professionnel” » un ensemble flou, segmenté, en constante évolution, regroupant des personnes exerçant une activité ayant le même nom doté d'une visibilité sociale et d'une légitimité politique suffisante sur une période significative » (1999, p.52).

Reprise par Demazière et Gadéa, (2009) cette notion met en évidence notamment la socialisation des professions dans le monde professionnel et son potentiel d'adaptation. La constatation des auteurs indique combien la visibilité installe la profession. L'enjeu en est sa délimitation. La difficulté de l'autonomie des groupes professionnels dépend de leur régulation interne et se situe au niveau de leur reconnaissance publique. Des tensions identitaires en animent souvent leur dynamique de construction. Deux points de vue sociologiques sur l'analyse de construction des professions en expliquent ces tensions: le fonctionnaliste et l'interactionniste. Longtemps antagonistes, ces deux approches anglo-saxonnes des professions nous intéressent car elles définissent pour la première un groupe professionnel relativement à ses normes (histoire, formation, diplômes, dénominations) alors que la seconde s'attache à un processus biographique et identitaire. Loin d'en faire la synthèse, la sociologie des groupes professionnels introduit néanmoins la réflexion sur des valeurs morales mais aussi symboliques ainsi que sur le contenu du métier (Champy, 2012). Dans la lignée de cette réflexion, Boussard, Demazière, Milburn, (2010) radicalise la notion de professionnalisation, les auteurs considèrent qu'elle répond désormais à une injonction. Cette injonction porte sur une définition précise de l'emploi dont les fiches de postes sont une 
figuration. En fin de compte, ce débat sociologique renvoie à l'effort de délimitation d'un groupe professionnel, laquelle passe nécessairement par la notion de compétences.

Le sociologue Zarifian définit la compétence - et non les compétences - comme «prise d'initiative (...) intelligence pratique des situations qui s'appuie sur des connaissances (...)» (Zarifian, 1999, p. 70). La prise d'initiative qu'il relie à la responsabilité constitue un des points forts de la compétence pour autant qu'un espace de liberté-créativité soit accordé à l'acteur professionnel par le management.

Plus récemment les chercheurs en sciences de l'information et communication se sont attachés à mettre en évidence la problématique identitaire dans la recherche d'une spécificité des métiers naturellement ceux de la communication. Ces recherches en SIC ont fait émerger les réflexions sur les compétences communicationnelles à l'heure du numérique plus particulièrement dans le domaine de la communication organisationnelle (Lépine et al., 2014). Les auteurs adoptent une posture scientifique précise issue de la volonté d'être empirique, elle «(...) consiste à considérer que ce sont les professionnels qui détiennent une connaissance incarnée par leur pratique » (Lépine et al., 2014, p. 6). Cette assertion fait écho à notre réflexion sur le processus d'apprentissage pris dans un dispositif universitaire. Les acteurs en co-présence participent selon leur positionnement dans et par le dispositif à la construction des compétences voulues, supposées, affichées des étudiants futurs communicants. Le dispositif contraint et d'une certaine façon euphémise les effets de tensions entre les acteurs dont la nature réside dans le processus de professionnalisation et in fine la construction du groupe professionnel des communicants. Un détour théorique par la notion de dispositif apporte des éléments de compréhension à ce processus.

Dans le cadre d'un entretien pour la revue Ornicar, Foucault en offre une définition utile qui sera reprise quasi-systématiquement et souvent dénaturée décontextualisée - par nombre de chercheurs (Cf. le chapitre de I. Gavillet, 2010, pp. 17-38 ${ }^{120}$ ). Le dispositif dit-il est

un ensemble résolument hétérogène, comportant des discours, des institutions, des aménagements architecturaux, des décisions réglementaires, des lois, des mesures administratives, des énoncés scientifiques, des propositions philosophiques, morales, philanthropiques, bref : du dit aussi bien que du non-dit [...]. Le dispositif lui-même, c'est le réseau qu'on peut établir entre ces éléments. (Foucault, 1977, p. 64).

\footnotetext{
${ }^{1}$ Gavillet Isabelle, (2010), Michel Foucault et le dispositif: questions sur l'usage galvaudé d'un concept, in Les dispositifs d'information et de communication, Concept, usages et objets, sous la direction de V. Appel, H. Boulanger, L. Massou, Bruxelles : De Boeck, chapitre 2, pp. 17-38.
} 
Mais l'hétérogénéité de ses éléments constitutifs ne suffit pas à « faire dispositif ». Foucault propose de repérer «(...) la nature du lien qui peut exister entre ces éléments hétérogènes (...) discursifs ou non » (Gavillet, 2010). En effet, le dispositif " sorte de formation » répond à une urgence à un moment donné et de ce fait prend une «fonction stratégique dominante»(Gavillet, 2010). Enfin le dispositif est à resituer comme mode de gouvernance stratégique de l'action. Un dispositif installe donc certaines relations de pouvoir entre les formations sociales qui le composent. La question du pouvoir est fondamentale, Foucault le prend dans son acception large, de la coercition à la protection. Le pouvoir, «c'est en réalité des relations, un faisceau plus ou moins organisé, plus ou moins pyramidalisé, plus ou moins coordonné de relations... » Gavillet, 2010). Agemben, dans le sillage de Foucault, pense que le schéma actanciel du dispositif d'apprentissage instaure certains rapports de force et idéologiques (Agemben, 2007). Le dispositif foucaldien, revu par Agemben intègre la notion des modes d'existence liée à des dispositifs techniques et reprend l'idée de la gouvernance de l'action dans une stratégie que le dispositif porte. Cet aspect technique du dispositif est le plus souvent mobilisé. Le philosophe le définit de la façon suivante, « j'appelle dispositif tout ce qui a, d'une manière ou d'une autre, la capacité de capturer, d'orienter, de déterminer, d'intercepter, de modeler, de contrôler et d'assurer les gestes, les conduites, les opinions et les discours des êtres vivants ». (Agemben, 2007, p. 12). L'auteur y décrit le sujet comme spectral, désubjectivé, dépossédé, soumis.

\section{Observation du dispositif d'apprentissage d'un Master en communication : formation et profession en construction}

Selon une approche compréhensive, nous proposons de mettre en corrélation les différents éléments qui composent le dispositif d'apprentissage : l'actualité de construction d'un groupe professionnel des communicants, l'organisation de l'apprentissage et ses normes, le fonctionnement du Master en communication posent les bases du cadre. Les acteurs, étudiants, professionnels, enseignants se voient une place assignée dans ce dispositif et contribuent à sa mise en sens. Nous avons proposé aux étudiants d'évaluer l'adéquation des enseignements aux activités professionnelles. Nous les présentons sous forme synthétique. Dans le même temps nous avons recueilli et synthétisé des données issues des échanges avec les professionnels-tuteurs hors de deux groupements en janvier et en juin. Cette dynamique est susceptible d'élucider la façon dont se construit le groupe professionnel de communicants, la place qu'y tient la formation initiale, l'expérience de terrain et les pratiques des professionnels. 
186 Le communicateur bousculé par le numérique

Notre état de la formation des étudiants en communication en situation d'apprentissage nous place d'emblée face à des préalables incontournables. D'un côté, les étudiants sont formés pour utiliser sur le terrain les approches théorisées de la communication organisationnelle et son marquage par le numérique. D'un autre côté, les professionnels qui les reçoivent et les accompagnent occupent donc une place capitale. Or certains éléments seront à prendre très au sérieux pour notre recherche :

- la communication dans les organisations de toutes natures reste une fonction essentialisée tout comme la gestion ou la comptabilité et qu'il en est attendu des effets mesurables en termes de visibilité et de qualité ;

- La matérialité de la communication est partiellement résolue par l'apparition et l'utilisation des outils numériques ;

- L'importance du terrain est à la base partagée entre tous les acteurs dans le cadre d'une co-construction-apprentissage.

\subsection{Une actualité utile du métier de communicant}

«Enseigner la communication, former à la communication, apprendre à communiquer... Est-ce effectivement réalisable?» (Benoit, 2000, p.9) s'interrogeait-on dès la fin des années 1990 en France. Durant cette période les professionnels de l'information et de la communication, certains formés à cette fonction d'autres non, avec ou sans titre, exerçaient dans les organisations et institutions. Jean-Luc Michel (1999) recensait déjà au début des années 2000, 280 fonctions afférentes à la communication et 60 métiers; cet éclatement marque l'étendue des possibilités d'activités du métier de "communicant» et la difficulté d'en circonscrire les compétences attendues. D'autant plus que les métiers de et dans la communication ont évolué, se sont précisés notamment relativement au numérique. L'émergence d'une profession qui répondait à certains besoins dans les entreprises déclencha l'apparition d'un grand nombre de diplômes « en communication » durant la première décennie des années 2000. Ceux-ci tentaient et tentent encore d'adapter les connaissances en info-communications des étudiants à la mutation des environnements et des formes organisationnels (Lahlou, 2000, Boutaud et al., 1998) sous l'effet des crises, de la mondialisation et d'Internet (Aglietta, 2010).

Plusieurs questions restent néanmoins en suspens: celle de la pratique des communicants et de leur formation et ses modalités de même que leur adéquation aux réalités des organisations et aux pratiques professionnelles. En effet, la légitimité des communicants opérationnels dans les organisations mettant en jeu leurs compétences, leur performance et leur efficience réputées difficiles à évaluer, est posée de façon récurrente (Michel, op.cit.). Nécessairement cette légitimité ne sera pas «donnée » aux étudiants en formation. Dans la mesure où les opérationnels 
ont gagné et gagnent une reconnaissance au fur et à mesure de leur pratique, les jeunes diplômés sont reçus de façon plus circonspecte notamment lorsqu'ils sont apprentis. Pourtant la mise à l'épreuve du terrain reste l'étape la plus indispensable pour gagner leur crédibilité. Le « stage » est la formule la plus classique de mise en contact avec l'entreprise mais le dispositif d'apprentissage participe de façon réelle à cette mise à l'épreuve en rapprochant un professionnel en poste et un étudiant salarié.

\subsection{Le dispositif d'apprentissage}

Le dispositif de formation par apprentissage est particulier car l'apprenti a un contrat de travail et la formation est organisée autour de « séjour en entreprise et de séjour à l'université » (Guès, 2010). L'apprentissage en tant que dispositif juridique en effet, relie dans le dispositif règlementaire, un centre de formation pour apprentis (CFA) qui délègue par un contrat avec l'université, l'enseignement dit théorique, pour des étudiants-apprentis qui sont donc salariés à mi-temps dans une entreprise sous la responsabilité d'un tuteur d'apprentissage. Dispositif humain, l'apprentissage instaure des relations entre un tuteur professionnel en communication en entreprise, l'enseignant responsable du diplôme, un étudiant-apprenti élément interface de la formation théorico-pratique. Dispositif topographique, l'apprentissage est composé de lieux hétérogènes, l'université et l'entreprise, qui auraient pu et/ou peuvent rester étrangers l'un de l'autre. Il est vrai que les discours respectifs sont réputés éloignés pour l'un des réalités de terrain, pour l'autre quelquefois des connaissances théoriques sur la communication et le plus souvent sur les impératifs universitaires. La notion d' «accompagnement» issue de l'esprit des «compagnons » y est fondamentale.

Le dispositif de l'apprentissage qui existe depuis la Révolution concernait les jeunes artisans.

La formation par apprentissage est juridiquement une formation initiale mais elle a deux spécificités qui l'éloignent de l'enseignement scolaire : l'apprenti a un contrat de travail signé avec un patron, ce qui lui donne des droits et implique des devoirs, la formation est organisée autour de séjour en entreprise et de séjour dans un Centre de formation d'apprenti (CFA) ». (Guès, 2010).

Ces centres de formation d'apprentis furent créés en 1966 en France. Le mémorandum du 27 mai 1986 précise que

L'apprentissage est un véritable système de formation professionnelle à temps plein associant deux lieux de formation: l'entreprise et le centre de formation d'apprentis. Il réunit autour d'un projet éducatif commun tous les acteurs concernés: jeunes, familles, entreprises, centres de formation, organisations professionnelles, compagnies consulaires, Régions, Etat et rajoutent 
188 Le communicateur bousculé par le numérique

l'apprentissage permet d'acquérir une formation générale et professionnelle sanctionnée par un diplôme de l'enseignement technologique et s'appuyant sur la pédagogie de l'alternance. (Id. Ibid).

L'apprentissage se déploie dans le supérieur en France dans cet esprit. La fin de ces études salariées est sanctionnée par un diplôme universitaire ${ }^{121}$ d'une valeur identique à un diplôme classique.

\subsection{Les enseignements du Master 2 CCOSII à l'UNS : Esprit et contenu}

Un des derniers Masters en communication intitulé Communication, cultures, organisation, stratégie d'images et Internet (CCOSII), créé en 2012, à l'Université de Nice Sophia Antipolis (UNS) se situe dans une forme résolument ancrée dans les pratiques des professionnels par la médiation de l'apprentissage. Ce dispositif de formation s'est élaboré d'une part sur des négociations préalables avec les organisations, d'autre part sur un enseignement académique de la communication organisationnelle, en grande partie puisé dans les sciences de l'information et de la communication $^{122}$. Il tente en fait de résoudre la tension entre un prescriptif d'origine entrepreneuriale et un réflexif d'ordre universitaire, par la co-construction de compétences renouvelées pour permettre d'élaborer des stratégies de communication ciblées et performantes pour l'organisation. La première année de Master en info-communication (Master 1) par apprentissage, ouverte en 2001, voit son programme pédagogique historiquement arrimé au Master classique. Une seule option (obligatoire pour les apprentis mais ouverte aussi aux étudiants non apprentis) offre l'aperçu d'une spécialisation en communication organisationnelle. La deuxième année de ce Master, ouverte en 2012, a pu prendre de ce fait toute son importance dans la professionnalisation d'autant plus qu'elle a bénéficié du retour d'expérience des étudiants-apprentis de Master 1. Notre étude porte uniquement sur cette deuxième année.

Les enseignements de ce Master se retrouvent dans son intitulé et dans les intentions de sa conception. Ils consistent à associer la connaissance des organisations, de la communication interne, marketing, publicitaire et événementielle tant du point de vue technique (enseignements des techniques logistiques et numériques) que conceptuel (enseignements théoriques) ${ }^{123}$ et

\footnotetext{
${ }^{121}$ Cf. http://www.onisep.fr/Choisir-mes-etudes/Apres-le-bac/Filieres-d-etudes/L-alternance-dans1-enseignement-superieur2/Alternance-dans-1-enseignement-superieur-etat-des-lieux

$1221 / 3$ des enseignants sont des titulaires du Département des Sciences de l'information et de la communication.

${ }^{123}$ Depuis 2012, dans la formation universitaire dans le Master 2 s'est orientée vers :
} 
stratégique (anticipation des besoins en communication). L'enseignement de l'essor et l'importance du numérique dans les mutations de la communication est résolument un parti pris de ce Master. Dès lors, la promesse contenue dans ce Master CCOSII est d'apprendre le métier de communicant relativement aux impératifs des organisations à l'heure de la révolution numérique généralisée, du web collaboratif et de la mondialisation des échanges.

Les paradigmes de la communication organisationnelle désormais définis dans le champ des sciences de l'information et de la communication sont à éprouver au contact des pratiques des professionnels en situation. Si le socle épistémologique enseigné aux étudiants permet d'appréhender la logique des systèmes enchevêtrés, la construction des territoires numériques et la mutation des temporalités et des spatialités, les narrations et la performativité des discours managériaux, il est réévalué à l'aune des pratiques confrontées au contexte de crise, d'évaluation et d'innovation forcée par la compétitivité accrue. De ce fait, l'enseignement ne s'associe pas simplement à un «supplément» numérique mais l'usage d'Internet vient orienter, reformer et réformer la communication organisationnelle dans sa structure même, ses objectifs, ses enjeux. Dans ce Master, le groupe professionnel des communicants est considéré comme le moteur du système organisationnel. Ils sont préparés pour gérer et façonner la structure appréhendée comme une totalité en action, une réalité où la combinaison d'une double compétence communicationnelle et stratégique s'impose.

\subsection{Des étudiants en construction professionnelle}

Méthodologiquement, nous avons travaillé avec la deuxième promotion des étudiants-apprentis du Master 2 selon une démarche compréhensive. Notre propre position d'enseignant-chercheur responsable du diplôme étudiant le dispositif a nécessité une prise de distance et un regard sans concession sur son fonctionnement. En effet, si « le chercheur doit s'immerger dans le contexte pour comprendre le point de vue des acteurs, il doit cependant garder une certaine distance par rapport à

- la communication organisationnelle enseignée selon ses approches et enjeux (tendances épistémologiques fonctionnaliste, systémique, symbolique, critique et constitutive).

- L'histoire des organisations et des relations au travail montrent que l'organisation est un « tissu de communications » (Van Every, Taylor).

- La philosophie du numérique et des transformations profondes des individus, des collectifs, des modes d'existence pour un regard critique sur l'émergence de nouvelles subjectivités (Noyer-Carmes) et socialités (Casilli).

- La stratégie de communication avec la mise en pratique du plan de communication (Libaert), de l'audit des outils de communication numérique et classique. 
son objet. » (Charmillot, Dayer, 2007, p.126) ${ }^{124}$. Nous nous inscrivons dans la ligne des travaux en recherches qualitatives qui privilégie l'approche des phénomènes sociaux en termes de transaction sociale.

La notion de transaction sociale implique une posture méthodologique exigeante dans la mesure où l'approche qu'elle génère présuppose une prise en charge conceptuelle de la complexité du social: organisation sociale, production de sens et historicité sont inter-reliés. Il s'agit dès lors, sur le plan de l'opérationnalisation, de tenir compte de différents niveaux d'analyse: transaction en soi (recherche de cohérence biographique), transaction entre soi et autrui (dans le cadre de la situation), et transaction entre ceux qui sont engagés dans le cadre de la situation et les environnements qui leur sont extérieurs. L'étude d'une transaction sociale implique par ailleurs la prise en compte de son déroulement dans le temps. Centrée sur un changement "en train de se faire", la transaction sociale est approchée par le biais de séquences chronologiques dont la succession est à lire en termes d'agir communicationnel (Charmillot, Dayer, 2007, p.134).

Notre population d'étude est constituée des vingt étudiants qui forment l'effectif des apprentis du Master 2. Elle est fortement féminisée avec seize étudiantes pour quatre étudiants (entre vingt-trois et vingt-cinq ans). Ils sont tous embauchés dans des entreprises de la région du Sud-est de la France.

Le recueil de nos données s'est effectué à partir des rapports des deux séances d'entretiens en présence du collectif à la fin des deux semestres (fin décembre et fin avril), des évaluations qualitatives écrites des cours et des demandes orales de compléments de cours et les raisons de ces demandes. Les données que ces documents fournissent, ont été réorganisées en thématiques significatives de la confrontation des enseignements et du terrain, de l'expression des manques et des incompréhensions des étudiants vis à vis de certains enseignements, des difficultés et des succès rencontrés dans l'entreprise.

Le compte rendu des catégorisations des données s'est imposé car des redondances apparaissaient de façon évidente et nous extrayons de l'analyse croisée des entretiens, des évaluations et demandes des étudiants les éléments centraux que nous présentons. Les notes des échanges des réunions qui firent l'objet de deux rapports validés par les étudiants et les enseignants présents constituent les perceptions des étudiants à propos de leur efficacité dans les organisations. Les évaluations des enseignements permettent entre autres réflexions des commentaires sur l'adéquation des cours aux pratiques de terrain. Les demandes de compléments

\footnotetext{
${ }^{124}$ Charmillot M., Dayer C. (2007) Démarche compréhensive et méthodes qualitatives : clarifications épistémologiques In recherches qualitatives - Hors Série - numéro 3, Actes du colloque bilan et prospectives de la recherche qualitative, http://www.recherchequalitative.qc.ca/documents/files/revue/hors_serie/hors_serie_v3/Charmillot_et_DayerFINAL2.pdf.
} 
de cours de certains étudiants mettent en évidence conjointement leurs manques relativement au service de communication dans lequel ils travaillent mais aussi le manque de connaissances notamment numériques de certains professionnels.

Les récurrences thématiques qui ressortent nettement de ces trois catégories de données permettent de tirer quelques conclusions certes provisoires - une étude longitudinale précisera dans les années qui viennent s'ils nous est possible de les confirmer - mais néanmoins pertinente sur un état d'une actualité de la profession appréciée par les futurs professionnels et des nécessités pour y accéder.

- L'expérience de terrain par l'apprentissage. La plupart des étudiants confirment que sur des terrains aussi diversifiés, la réflexion à partir des enseignements ne peut prendre corps qu'au contact direct avec un communicant en activité. Les apprentis considèrent que l'implication et la mise à l'épreuve ne sont jamais autant sollicitées que lorsque l'étudiant appartient pleinement au groupe des salariés. A ce titre le principe de l'apprentissage est exemplaire dans la mesure où l'étudiant est considéré comme le salarié d'une organisation durant une ou deux années. Pourtant le dispositif tend à donner au tuteur une importance notable dans l'ensemble de la formation (deux visites aux entreprises sont effectuées dans l'année lors desquelles le tuteur présente sa pratique et ses apports à l'apprentis) et dans l'accompagnement de l'étudiant apprentis. Un tiers des étudiants peut prendre des initiatives, travailler en responsabilité et l'avis de deux d'entre eux est écouté par l'équipe de direction ou le tuteur.

- Le pouvoir de l'utilisation des outils numériques. Les trois quarts des étudiants valorisent les enseignements techniques numériques et ils considèrent qu'ils sont insuffisamment enseignés tout au long du cursus SIC depuis la licence. Leur demande de formations supplémentaires porte sur la technique essentiellement. En effet, ils constatent que leurs compétences techniques sont fortement sollicitées par les tuteurs soit pour compenser le manque de connaissance de ces derniers soit pour autonomiser l'étudiant sur une tâche précise de réalisation numérique. Toute communication interpersonnelle et externe passe par les outils numériques (plateforme collaborative, réseaux professionnels pour le recrutement, création d'évènement) pour lesquels une expertise des étudiants est attendue.

- Apprendre la ou les stratégie(s) communicationnelle(s). Les étudiants pensent que les enseignements qui traitent de stratégie communicationnelle ne leur permettent pas de comprendre les enjeux de la communication, d'anticiper les besoins des entreprises comparativement à leur activité sur le terrain. Ils participent peu aux réunions de direction et ne sont informés que par leur tuteur. Pourtant l'aspect stratégique ne s'acquiert qu'auprès des professionnels selon les étudiants. Les étudiants-apprentis affirment se construire professionnellement en construisant leur projet professionnel et en rédigeant leur mémoire professionnel. De façon générale, si la mise en relation du contenu 
192 Le communicateur bousculé par le numérique

des enseignements et de la pratique parait difficile, ils le réfèrent aux objectifs et orientations imposées par le terrain. Celui-ci est spécifiquement formateur selon les besoins, les urgences, les connaissances du tuteur professionnel. C'est la raison pour laquelle les fonctions confiées aux étudiants-apprentis restent mal définies dans la mesure où les responsables communicants ne savent pas décrire leurs propres pratiques. Les étudiants se définissent eux-mêmes « community manager, ressources humaines, évènementiels, assistante de communication interne, animateur multimedia, etc. ».

Généralement les étudiants constatent que leurs connaissances des techniques numériques sont parcellaires, éclatées et instables. Ils ne prennent pas part aux décisions et stratégies managériales. Enfin ils entretiennent des relations proches avec le tuteur, lequel le plus souvent, utilise les compétences numériques des étudiants.

Ces quatre exemples de sujet de mémoire de fin d'année montrent la diversité des trajectoires d'étudiants, la place du numérique, la spécificité organisationnelle et la façon dont se dessine la profession de communicants.

- - La création d'une communauté virtuelle d'experts dans la branche médicale de Siemens Healthcare France (tuteur =ingénieur)

- De l'évolution de l'image du chef pâtissier et de ses nouveaux besoins communicationnels en milieu artisanal, Le Cas de Mic Mac Macaron (tuteur = pâtissier)

- Stratégie de communication digitale et industrie pharmaceutique. Étude du laboratoire pharmaceutique vétérinaire Virbac (tuteur $=$ chef du personnel)

- Nouvelles formes de communication 2.0, étude de cas de la plateforme ecobiz 2.0/ CCI Nice Côte d'Azur (tuteur: président de la CCICA)

Le tuteur n'est donc ni nécessairement un communicant, ni un diplômé en communication du supérieur. Pourtant, la plasticité des apprentis et la volonté des d'accompagnement des tuteurs crée un binôme efficace pour les deux parties.

\subsection{Les entreprises d'accueil : positionnement des professionnels}

Deux réunions des maîtres d'apprentissage nous ont permis de constituer ce groupe de professionnels comme un groupe focus. Leurs échanges se répartissent dans l'analyse qui suit et qui reprend les points de vue et de vécus communs des communicants en poste, tuteurs d'apprentissage, sur une définition de leur profession, de leur relation avec les apprentis et un point de vue sur leurs connaissances académiques. 
Les maîtres d'apprentissage sont responsables de communication en charge de la gestion du personnel, de l'externe, de l'événement mais encore responsable d'un service généraliste où toute action de communication se fait, technique ou de relations humaines. Ils sont rarement diplômés spécifiquement en communication (deux sur vingt-quatre ont un Master en communication des organisations de l'Institut d'administration des entreprises). Ils ont entre trois ans et douze ans d'ancienneté sur le poste et sont dans une tranche d'âge de trente-cinq à cinquante ans. Ces professionnels occupent des postes spécifiques sans nécessairement avoir reçu de formation spécifique en communication. Ils utilisent une connaissance expérientielle souvent technique et commerciale, et gagnent des savoirs faire qu'ils ne savent souvent pas décrire mais qu'ils savent transmettre. Les savoirs qu'ils délivrent sont dépendants des formes organisationnelles et du milieu professionnel, ils s'y sont adaptés pour conserver leur poste et forment l'apprenti dans le même état d'esprit d'adaptation aux besoins immédiats de l'organisation.

Les organisations associées sont des agences de publicité-marketingcommunication interne, des grandes et moyennes entreprises et de très petites entreprises (TPE), des associations et des institutions (collectivité locales et territoriales). Les entreprises offrent un panel varié des lieux d'apprentissage, agence de communication, mairie, grande distribution, grandes entreprises du transport, de la gestion de l'eau ou de l'électricité (Véolia), opérateurs mobiles (Orange), entreprise du médicament, etc.

- La mise en place des enseignements tient compte du fait que les organisations qui reçoivent ces apprentis, baignent inégalement dans la culture numérique et la gestion informationnelle. Pour certaines de ces entreprises, l'utilisation des technologies de la connaissance, de la communication et des réseaux sociaux parait souvent relever de la nécessité ou de la mode plus que d'une vraie stratégie managériale du numérique. Pour autant ils ne prennent pas en compte les contenus des enseignements dont ils sont pourtant informés.

- Les organisations ont saisi qu'à travers l'usage du numérique leur image se trouve mobilisée qu'elle soit en affectée ou valorisée. Nécessairement la communication interne et externe subit des mutations structurelles dont les effets immédiats sont de recomposer sans cesse l'image de l'entreprise sans nécessairement la maîtrise souhaitée par le management. Les responsables de communication mettent en place la stratégie interne et externe au moyen d'évènements internes dont la préparation numérique échoit à l'apprenti.

- Ils ont la charge de la veille informationnelle dans le domaine de gestion de crise ou de concurrence. Mais il ne donne pas accès à ces deux domaines trop sensibles aux apprentis, les enjeux pour l'entreprise sont jugés trop déterminants. 
194 Le communicateur bousculé par le numérique

- Ils ont la charge d'encadrer et animer le travail collectif et mettent en place des outils numériques collaboratifs, des réseaux sociaux d'entreprise dont l'apprenti assure l'animation. Ils sont en charge de l'image marketing de l'organisation, l'apprenti participe à l'infrastructure organisée par des agences extérieures dont l'interlocuteur direct est le responsable de communication. Bref, l'apprenti est le délégataire de la surcharge de travail du responsable communication, et n'a que très rarement la responsabilité d'une mission dédiée.

- Dans certaines entreprises au contraire, le numérique apparaît déjà au centre des communications professionnelles. Leur communication organisationnelle s'enrichit et se complexifie avec le numérique. Sa technicité doit être assumée par les communicants selon de nouvelles modalités d'être et de faire dans des relations renouvelées aux objets techniques, aux territoires, aux identités (la leur et celle de leur entreprise). Leur adaptation est fondamentale pour la reconnaissance qu'ils reçoivent de leur structure. De ce fait, les tuteurs ne délèguent pas leurs responsabilités, trop attachés à légitimer leur poste.

Ces professionnels apprécient le dispositif de l'apprentissage. En effet, l'assistance d'un apprenti leur est fort utile car ils savent lui attribuer des tâches complémentaires et utiles pour leurs activités. L'accompagnement qui s'opère est d'ordre technique (positionnement pertinent sur une mission technique) et humain (attention portée aux difficultés de l'apprenti). Ce fonctionnement en binôme est apprécié car il est dit construire et accroître la confiance mutuelle. Il a été noté par tous que la possibilité - occasionnelle - de libérer l'apprenti de ses cours pour participer à un évènement important pour l'entreprise conforte les responsables dans l'importance de la formation par accompagnement direct. Les enseignements numériques attirent leur intérêt alors qu'ils pensent que les autres enseignements ne sont intéressants que pour la «culture générale » des étudiants apprentis.

Le potentiel de formation du terrain est survalorisé par les étudiants (pas d'absentéisme en entreprise), les professionnels (dérogation pour mobiliser un étudiant pendant ses cours) et d'une certaine façon le dispositif d'apprentissage tout entier. Celui-ci dans son histoire-même s'est forgé la représentation forte du compagnonnage efficace pour un "professionnel exemplaire» en termes de formation et d'employabilité. Les enseignants et les responsables du Master cautionnent ou se soumettent et admettent que le terrain est plus formateur que l'enseignement académique.

\section{Les perpétuelles mutations des communicants, formation en mutation, profession en construction}

Le cadre du dispositif d'apprentissage installe des normes de fonctionnement qui lient les composants actifs pour construire des compétences et permettre l'insertion 
professionnelle : savoirs universitaires enseignés, savoir-faire en organisation transmis et trajectoire d'un étudiant-apprenti. S'entremêlent dans le dispositif d'apprentissage en communication des pragmatiques de discours, de techniques, d'idéologie des enseignants et des professionnels générant des tensions agonistiques que les étudiants gèrent selon leurs objectifs personnels. Les forces à l'œuvre dans ce que nous appelons le dispositif d'apprentissage, mettent en jeu des groupes sociaux institués (université, entreprise, groupe des communicants, groupe des étudiants), hétérogènes dont il convient d'apprécier la stabilité, les évolutions, les objectifs individuels et collectifs. Un communicant gagne en crédibilité par ses compétences sur le terrain et de ce fait la même épreuve est imposée aux apprentis. Le diplôme demeure le prétexte (non négligeable certes) à une possible embauche mais la stabilité de l'emploi reste suspendue à l'efficacité sur le terrain et cette efficacité passe désormais par la maîtrise des outils numériques. Le groupe professionnel des communicants nous semble en reconstruction, remis en question par l'arrivée massive du numérique.

De façon générale, les compétences communicationnelles pour être actualisées, doivent prendre en compte la mutation des organisations sous la pression économique croissante et insistante. À ces transformations socio-économiques s'associe l'installation à marche forcée du numérique dans toutes les sphères de la vie privée et professionnelle. Ce contexte crée une confusion continue dans l'appréciation des compétences requises. En effet, certaines sont apprises (connaissance de logiciels), d'autres acquises intuitivement (Internet et les réseaux sociaux) dans le bain du numérique et ses objets connectés notamment pour les générations $\mathrm{Y}$ à laquelle correspondent des étudiants actuels. Les formations spécifiques à la communication ont l'obligation de préparer les étudiants à ces mutations organisationnelles en relation avec le numérique (De Conninck, 2006, Mallard, 2009, Smoreda, 2002). Les étudiants arrivent dans les organisations avec des niveaux inégaux de connaissance numérique mais tous sont inscrits sur les réseaux sociaux dont ils usent avec une habileté relative.

Les futurs communicants en formation tentent de traduire sur le terrain les approches théorisées de la communication organisationnelle modifiée par le numérique. Mais le plus souvent une adaptation rapide et efficace s'impose pour répondre au plus vite aux besoins des organisations et du tuteur. Dans cette mesure il peut y avoir une certaine violence dans le dispositif au niveau de la redistribution du pouvoir. En effet, le dispositif remet sans cesse en question le processus d'échange pour l'ajuster aux objectifs finaux collectifs. Dans le cas de l'apprentissage, le but quasi-unique est l'employabilité. Nous constatons que le dispositif d'apprentissage donne la primauté aux terrains et à leurs contextes organisationnels spécifiques. Dans le processus de professionnalisation, l'adéquation des théories mobilisées dans les enseignements à la réalité des pratiques de terrain intervient moins que la dynamique de relation entre le tuteur et l'apprenti. Une certaine soumission de 
l'apprenti à cet ensemble organisationnel figuré par le tuteur marque cette relation. Cette forme de coercition du dispositif pour paraphraser Agemben (cf. supra) s'accompagne d'une réorientation de la stratégie générale. Cette stratégie finalement suit les évolutions de la profession de communicants que la diversité du numérique « éclate» de nouveau en une constellation d'expertises possibles.

L'actualité de la construction des métiers de la communication montre que le métier subit une évolution constante dès le début des années 2000. Cette évolution est typique de l'effort de construction d'un groupe professionnel. Malgré un effort notable, les communicants ne forment toujours pas un groupe professionnel identifié dans la mesure où les professionnels sont encore recrutés parmi les salariés de l'organisation pour leur sensibilité à la communication et/ou leur ancienneté dans l'entreprise ou l'institution. Cette situation se poursuivant, les communicants sont en transformation constante et stabilisent leur poste plus que leur profession. De ce fait, le dispositif de l'apprentissage dont la norme imposée est l'équivalence entre le temps en entreprise et le temps en université, se plie à une prédominance du terrain sur la formation. Le pouvoir est devenu asymétrique, le primat du terrain accroit l'essentialisation de la communication dans les organisations par tous les acteurs professionnels, étudiants, enseignants. Tous se trouvent «librement» soumis aux pressions managériales pour que leur travail soit d'une efficacité mesurable. La déperdition du réflexif au profit des réalisations numériques est un effet collatéral des mutations organisationnelles. L'efficience se joue dans le rapport à l'employabilité et sur ce plan le terrain donne ses critères d'évaluation et le projet professionnel et la rédaction du mémoire des étudiants en témoignent. La construction de la profession toujours en cours semble tendre à la rationalisation de ses activités, leur forte orientation au tout numérique et à la performance de la réalisation.

Le projet professionnel est un point de départ d'une mise en sens processuelle qui se poursuivra durant toute l'année d'apprentissage pour qu'il trouve le moyen terme entre les impératifs de l'organisation, de son maitre d'apprentissage et de ses désirs et ambitions. La rédaction d'un mémoire met en forme le projet professionnel, le précise, le densifie en connaissances théoriques et appliquées quant à l'approche de la complexité des situations de communications. Entre les enseignements théoriques et la formation de terrain dirigée, il y a le potentiel personnel qui fait les itinéraires et trajectoires individuelles. Il s'opère une sorte de pont entre l'université et l'organisation par l'opération de traduction de l'étudiant pour s'intégrer dans l'entreprise. Tel que l'indiquaient Boutaud, Bonnet, Livio (1998), il s'agit de favoriser la «professionnalité» de l'étudiant plus que le professionnalisme. «La professionnalité conjugue le développement d'une dynamique au service d'un projet personnel, avec la constitution de repères scientifiques et techniques durables et réinvestissables, susceptibles en cela de pérenniser une aptitude au changement et à la pensée de complexité » (Boutaud, Bonnet, Livio, 1998, p. 24). Le mémoire reste 
le seul moment et la seule activité lors desquels les maîtres d'apprentissage interviennent dans l'élaboration réflexive d'une problématique qui les concerne autant que l'étudiant.

A l'intérieur du dispositif se forment les compétences et peut être se transformeront-elles aussi dans les points de rencontres comme des granularités de la formation. Pour P. Zarifian, «La compétence est une intelligence pratique des situations qui s'appuie sur des connaissances » (1999, 70). Les connaissances se croisent assez classiquement entre les données théoriques proposées aux étudiants et celles des professionnels. Or les enseignements sont jugés « trop » théoriques car ils ne peuvent directement et immédiatement s'appliquer sur le terrain. Ils demandent à être engrammés en attendant leur actualisation même si ce temps ne correspond pas aux temps de l'urgence des organisations comme le disait Etchegoyen.

Cet état des lieux réalisé par les étudiants-apprentis marquent bien l'écart entre ce qui est prescrit, ce qui est réalisé, ce qui est réflexif. Par ailleurs, pour Le Boterf (1997) « la compétence est la mobilisation ou l'activation de plusieurs savoirs, dans une situation et un contexte donnés ». L'apprentissage témoigne cependant d'une actualité originale, cette co-construction des savoirs. Les compétences «en communication » s'installent dans ce nœud complexe de circulation des connaissances entre des acteurs et des techniques selon un accompagnement de terrain qui rétroactivement influence les enseignements académiques. Par ailleurs, Dubar, Tripier, Boussard (2011) confrontés à la polysémie afférente au terme de "profession», en montrent en référence aux approches sociologiques anglosaxonnes et françaises, son aspect éminemment culturel.

Trois éléments fondamentaux sont attendus dans la combinatoire du dispositif : savoirs universitaires enseignés, savoir-faire en organisation transmis, et un étudiant-apprenti-professionnel. Il semble que le compagnonnage des professionnels en activité et le niveau personnel des connaissances numériques des étudiants construisent en fait les compétences disons adhoc et permettent l'insertion professionnelle. Le destin du groupe professionnel des communicants actuellement fortement lié au développement du numérique reste encore en suspens plus proche d'un groupe de métiers de la communication.

Les résultats de la recherche indiquent que le processus de reconnaissance ne s'élabore que par la mise en valeur des réalisations pratiques au dépens des enseignements théoriques. Ce déséquilibre a ses effets positifs car il favorise l'installation de la confiance des étudiants et des enseignants avec les professionnels. C'est actuellement ce processus qui semble générer une forme de légitimité et de reconnaissance des acteurs universitaires (enseignants, étudiants) par les acteurs professionnels. Il contribue à renforcer leur forme, l'identité des communicants sans pour autant la stabiliser. 
Ainsi le dispositif au sens de Foucault n'est plus la structure, il est l'action et les moyens de l'action, une action stratégique qui visent la professionnalisation de futurs communicants dont le projet personnel et universitaire les a conduit à traverser, subir, s'approprier, modifier les savoirs et savoir-faire. Les étudiants puisent dans le dispositif le sens de leur formation qui les conduit à un métier et des compétences définitoires d'une identité professionnelle spécifique.

\section{Réflexions conclusives}

S'il est possible d'admettre que les professions de communicants traversent une crise et que "l'hypothèse est que la crise de la profession s'inscrit dans une crise sociétale, celle du sens du travail et de l'entreprise, impliquant pour la fonction communication une mutation profonde » (Lépine et al., p.6), la mutation nous paraît à vrai dire marquée spécifiquement par l'essor du numérique. En effet, comprendre la communication organisationnelle comme le paradigme d'une communication organisante suppose, au-delà des définitions de référence des universitaires, d'y combiner les réalités de terrains. Celles-ci sont ancrées dans l'accélération concurrentielle des organisations que suivent les agences de création ou de conseil et qui contraignent les communicants à une opérationnalité adaptative. Cette adaptation se traduit par un renforcement de l'aspect fonctionnel et techniciste des réalisations. La connaissance et la maitrise de la place de la communication dans les organisations (pendant l'apprentissage), la stabilisation du point de vue théorique autrement dit le point de vue préalable de l'utilité et l'efficacité de la communication (pendant les cours) et l'anticipation stratégique des actions et constructions communicationnelles (la créativité issue des relations entre les étudiants et les professionnels) fondent l'apprentissage du métier de communicant. Nous ne pensons plus alors à une crise de la profession mais à une mutation toujours en cours parce qu'aliénée à sa technique, à sa course à la maîtrise des techniques numériques supposée mettre en forme les informations les plus percutantes, persuasives dans le sens du marketing (Lépine et al., idem).

Par ailleurs un autre écueil se pose à la stabilisation d'une profession de communicant reconnue et recherchée par les entreprises : la connaissance du secteur d'activité organisationnel et des stratégies appropriées aux problématiques du microniveau (individu) et du meso-niveau (groupe). L'apprentissage parait résoudre partiellement ce réel problème car l'étudiant-apprenti passe dans l'organisation, un temps utile pour développer sa connaissance du milieu. Ce savoir est nécessaire à sa participation aux stratégies spécifiques organisationnelles. Enfin les logiques de communications numériques étendent le territoire organisationnel qui implique la maîtrise, la gestion, le stockage des données qui assurent en même temps la mémoire et l'extension organisationnelle d'une façon créative voire unique pour chaque organisation. Cette période est une transition, une opportunité aussi pour les 
communicants actuels et futurs pour aborder leur métier renouveler par le numérique. De ce fait les formations se complexifient pour assurer la combinaison techno-stratégique que requiert le management d'une « communication réticulaire ».

\section{Bibliographie}

Agemben, G. (2007). Qu'est-ce qu'un dispositif. Paris : Éditions Payot \& Rivages.

Aglietta, M. (2010). La crise, les voies de sortie. Paris :Michalon.

Benoit, D. (2000). Former à la communication est-ce véritablement possible ? Dans D. Benoit, P. Fayard et J-P. Gehin (dir.), L'impossible formation à la communication (p. 9-29). Paris : L'Harmattan.

Bourdoncle, R. (2000). Autour des mots : professionnalisation, formes et dispositifs. Recherche et formation, (35), 117-132.

Boussard, V., Demazière, D. et Milburn, P. (dir.) (2010). L'injonction au professionnalisme. Analyses d'une dynamique plurielle. Rennes : PUR.

Boutaud, J.J., Bonnet, J. et Livio, D. (1998). De l'analyse des processus à l'émergence d'un métier. L'expérience du DESS stratégie de communication internationale (Dijon). Les formations à la communication, Communication et organisation, (14). Repéré à http://communicationorganisation.revues.org/2080

Champy, F. (2012). La sociologie des professions. Paris : PUF.

De Coninck, F. (2006). Privé/professionnel, Convergences et divergences. Réseaux, 24(140), 11-25.

Demazière, D. et Gadéa, C. (2009). Sociologie des groupes professionnels, Acquis récents et nouveaux défis. Paris : La Découverte.

Dubar, C. (1991). La socialisation ; Construction des identités sociales et professionnelles. Paris: A. Colin.

Dubar, C., Tripier, P. et Boussard, V. (2011). Sociologie des professions. Paris : Armand Colin.

Dubar, C. (1999). Sociologie des groupes professionnels, un bilan prospectif. Dans M. Menger (dir.), Les professions et leurs sociologies : modèles théoriques, catégorisations, évolutions : actes du colloque de la Société française de sociologie (p. 51-60). Paris : Les Éditions de la MSH.

Dubar, C. et Tripier, P. (1998). Sociologie des professions. Armand Colin : Paris. 
200 Le communicateur bousculé par le numérique

Durampart, M. (2014). L'organisation apprenante face aux enjeux de l'intégration des dispositifs numériques. Dans S. Parrini-Alemanno (dir.). Communication Organisationnelle, Management et Numérique (p. 59-68). Paris : L’Harmattan.

Foucault, M. (1977). Le jeu de Michel Foucault, Ornicar? Bulletin périodique du champ freudien, (10), 62-93.

Lahlou, S. (2000). La cognition au travail et ses outils: débordement, révolution, distribution. Intellectica, 1(3), 7-17.

Le Boterf, G. (1997). Compétence et navigation professionnelle. Paris : Editions d'organisation.

Le Moënne, Ch. (1997). Communication et induction dans les démarches de recompositions organisationnelles : d'une logique de la vérité à une logique de la convenance, Communication et organisation, (12). Repéré à http://communicationorganisation.revues.org/1999

Lépine, V., Martin-Juchat, F., Millet-Fourrier, C. (2014). Acteurs de la communication des entreprises et organisations, pratiques et perspectives. Grenoble : Presses universitaires de Grenoble.

Lépine, V. (2015). Les mutations professionnelles à l'heure du numérique. Dans S. Parrini-Alemanno (dir.) Communication Organisationnelle, Management et Numérique (p.427-436). Paris : L'Harmattan.

Michel, J.L. (1999). Les professions de la communication. Fonction et Métiers. Paris : Ellipses.

Passeron, J.C. (2005). Le raisonnement sociologique: un espace non poppérien de l'argumentation (éd. Rév.). Paris : Albin Michel.

Smoreda, Z. (2002). Présentation, Cycle de vie et sociabilité, Réseaux, (115), 9-13.

Vézinat, N. (2010). Une nouvelle étape dans la sociologie des professions en France. Sociologie, 1(3). Repéré à http://sociologie.revues.org/517

Zarifian, P. (1999). Objectif Compétence. Pour une nouvelle logique. Paris: Liaisons.

Zarifian, P. (2009). Le travail et la compétence entre puissance et contrôle. Paris : PUF. 


\section{Webographie}

Guès, P. (2010). Histoire de l'apprentissage en France. Repéré à http://patrickgues.wordpress.com/2010/10/01/histoire-de-lapprentissage-enfrance/

Mallard, A. (2009, septembre). Agir avec les TIC entre les sphères privée et professionnelle, recherches Orange Labs, Actes du colloque ISD : dynamique des usages des systèmes d'Information-Premier état des lieux. Repéré à http://www.fondation-cigref.org/wp/wp-content/uploads/2010/06/

L_interaction_espace_prive-espace_public-A.-Mallard.pdf 